\title{
MicroRNA expression in human intestinal Caco-2 cells in response to a flavonoid-rich berry extract
}

\author{
S. H. M. Cheung, P. A. Sharp, V. R. Preedy and H. Wiseman \\ King's College London, Diabetes and Nutritional Sciences Division, Franklin-Wilkins Building, 150 Stamford Street, \\ London, SE1 9NH, UK
}

MicroRNAs (miRNAs) are short non-coding RNAs which regulate gene expression post-transcriptionally by triggering mRNA degradation or translation repression ${ }^{(1)}$. Little is known about the role of miRNAs in the gastrointestinal tract. They may be instrumental in regulating gene expression in intestinal epithelial cells, particularly in response to nutrients. The present study investigated miRNA expression in response to a flavonoid-rich berry extract ${ }^{(2)}$ in human intestinal Caco-2 cell monolayers.

Human intestinal Caco-2 cells, cultured for $19 \mathrm{~d}$, were treated for $16 \mathrm{~h}$ with a flavonoid-rich berry extract (Optiberry; Interhealth Nutraceuticals, Benicia, CA, USA) at a final concentration of $0.125 \%(\mathrm{w} / \mathrm{v})$. Subsequently miRNA was extracted, pooled and used for the miRNA microarray analysis (Affymetrix ${ }^{\circledR} ;$ UK) and validated by quantitative RT-PCR. MiRNA microarray data was analysed using the bioinformatic TargetScanHuman 5.1 database, which predicts miRNA targets.

The results showed that 162 miRNA species were detected $(P<0.01)$. Using quantile normalisation, 2 miRNAs were up-regulated and 27 were down-regulated in response to the berry extract. Previously we have shown that incubation with berry extract decreases expression of the intestinal sugar transporter GLUT2 in Caco-2 cells ${ }^{(3)}$. Bioinformatic analysis identified nine of these differentially expressed miRNAs as potential regulators of the intestinal sugar transporter, GLUT2. Validation by qRT-PCR showed that two of these miRNAs, let-7a $(P=0.001)$ and miR-106b $(P<0.001)$ were significantly up-regulated in Caco-2 cells in response to berry flavonoids.

Studies are in progress, using RNA interference techniques, to investigate whether let-7a and miR-106b directly regulate GLUT2 expression.

1. Chekulaeva M \& Filipowicz W (2009) Curr Opin Cell Biol 21, 452-460.

2. Zafra-Stone S, Yasmin T, Bagchi M et al. (2007) Mol Nutr Food Res 51, 675-683.

3. Alzaid F, Pourvali K, Sharp PA, Bagchi D, Preedy VR \& Wiseman H (2010) Proc Nutr Soc 69, E326. 